\title{
Tramadol as an Analgesic to Treat Chronic Pain: A Review
}

\author{
${ }^{1}$ Megha Das, ${ }^{2}$ Rajratna Sonone, ${ }^{3}$ Himanshu Thukral, ${ }^{4}$ Radhika Lamba, ${ }^{5}$ Swati \\ Arora, ${ }^{6}$ Mallika Sethi \\ ${ }^{1}$ BDS, ITS Dental College, Ghaziabad - India \\ ${ }^{2}$ MDS, Oral and Maxillofacial Surgeon, Pune - India \\ ${ }^{3} \mathrm{MDS}$, Oral and Maxillofacial Surgeon, New Delhi - India \\ ${ }^{4}$ BDS Tutor, Department of Oral and Maxillofacial Surgery, ITS Dental College, Greater NOIDA - India \\ ${ }^{5} \mathrm{BDS}, \mathrm{MPH}$ (Epidemiology) - India \\ ${ }^{6}$ MDS Reader, Department of Periodontics, ITS Dental College, Ghaziabad - India
}

\begin{abstract}
International Association for the Study of Pain (IASP) defines Pain as an unpleasant sensory and emotional experience associated with actual or potential tissue damage, or described in terms of such damage. Pain has now been equated to a fifth vital sign highlighting the significance of pain management in patient care. Tramadol is a centrally acting analgesic which is structurally related to codeine and morphine. It is effectively used to treat moderate to severe acute and chronic pain in diverse conditions. Tramadol is placed on the second step of WHO analgesic ladder and in contrast to traditional opioids, exerts its analgesic activity, a dual mechanism of action inhibiting transmission as well as perception of pain. Tramadol is more suitable than NSAIDS and coxib's for patient with GI, renal and cardiovascular problems. Combined with low dependencelabuse potential, it has proven to be of significant advantage over other agents especially in the elderly.
\end{abstract}

Keywords: Tramadol, Analgesic, Pain, Cyclo-oxygenase, Adverse Effects

\section{Introduction:}

Pain is part of the body's defense system, triggering mental and physical behavior to end the painful experience. Pain perception is the sum of complex sensory, emotional and cognitive processes. The International Association for the Study of Pain (IASP) defines pain as unpleasant sensory and emotional experience associated with actual or potential tissue damage or described in terms of such damage. In contrast to acute pain, chronic pain is not a protective response and is no longer linked to a stimulus. Remodeling within the central nervous system results in persistent pain, lowered pain threshold, perception of pain caused by the usually nonpainful stimuli and spread of pain to areas other than those involved with initial pathology ${ }^{1-3}$.

The most common etiology of chronic non-malignant pain is musculoskeletal such as arthritis, cancer; peripheral nerve disorders and diabetes are other conditions that cause chronic pain. Pain has been acknowledged as the fifth vital sign by the American Pain Society and pain assessment and treatment much now be integrated into overall patient management. The 3 -step analgesic ladder, originally proposed for cancer pain relief by the WHO is useful and now widely employed for all types of pain, including the chronic pain of musculoskeletal diseases ${ }^{\mathbf{4}, \mathbf{5}}$.

\section{Pain Pharmacotherapy:}

NSAIDSs, Opioids and co-analgesic (antidepressants, anticonvulsants and calcium channel blockers) form the mainstay of pharmacotherapy for chronic pain. But, their use is often limited by side effects ${ }^{6}$.

2.1. Non-steroidal Anti-inflammatory Drugs: Usually abbreviated to NSAIDS, are drugs with analgesic, antipyretic and in higher doses, with anti-inflammatory effects. The term non-steroidal is used to distinguish these drugs from steroids, which have a similar eicosanoid-depressing, anti-inflammatory action ${ }^{7}$.

2.1.1. Mode of Action: Most NSAIDS act as non-selective inhibitors of the enzyme cyclo-oxygenase, inhibiting both the cyclo-oxygenase-1 (COX-1) and cyclo-oxygenase-2 (COX-2) isoenzymes. Cyclo-oxygenase catalyzes the formation of prostaglandins and thromboxane from arachidonicac id. Prostaglandins act as messenger molecules in the process of inflammation ${ }^{8}$.

2.1.2. Adverse Effects: The widespread use of NSAIDS has meant that the adverse effects of these relatively safe drugs have become increasingly prevalent. These effects are dose dependent and in many cases severe enough to pose the risk of ulcer perforation, upper gastrointestinal bleeding and death limiting the use of NSAIDS therapy. An estimated 10-20\% of NSAIDS patients experience dyspepsia and NSAIDS associated 
upper gastrointestinal adverse events are estimated to result in 103,000 hospitalizations and 16,500 deaths per year in the United States and represent $43 \%$ of drug-related emergency visits ${ }^{9}$.

2.1.3. Cardiovascular Risk: A recent meta-analysis of all trials comparing NSAIDSs found an $80 \%$ increase in the risk of myocardial infarction with both newer COX-2 antagonists and high dose traditional antiinflammatory compared with placebo. NSAIDS aside from aspirin are associated with a doubled risk of symptomatic heart failure in patients without a history of cardiac disease. In patients with such a history, however, use of NSAIDS was associated with more than 10-fold increase in heart failure ${ }^{\mathbf{1 0}}$.

2.1.4. Gastrointestinal ADRs: The main ADRs (adverse drug reactions) associated with use of NSAIDS relate to direct and indirect irritation of the gastrointestinal tract (GIT). NSAIDS causes a dual insult on the GIT: the acidic molecules directly irritate the gastric mucosa, and inhibition of COX-1 reduces the levels of protective prostaglandins. Common gastrointestinal ADRs include Nausea, Vomiting, Dyspepsia, Gastric ulceration /bleeding and Diarrhea ${ }^{11}$.

2.1.5. Renal ADRs: NSAIDS are also associated with a relatively high incidence of renal adverse drug reactions. The mechanism of these renal ADRs is due to changes in renal hemo-dynamics, ordinarily mediated by prostaglandins, which are affected by NSAIDS. Prostaglandins normally cause vasodilatation of the afferent arterioles of the glomeruli. This helps maintain normal glomerular perfusion and glomerular filtration rate (GFR), an indicator of renal function. By blocking this prostaglandin-mediated effect, NSAIDS ultimately may cause renal impairment. Common ADRs associated with altered renal function include Salt and fluid retention and Hypertension. These agents may also cause renal impairment, especially in combination with other nephrotoxic agents. In rarer instances NSAIDS may also cause more severe renal conditions like interstitial nephritis, Nephrotic syndrome, acute renal failure and acute tubular necrosis ${ }^{12,13}$.

2.1.6. Photosensitivity: Photosensitivity is a commonly overlooked adverse effect of many of the NSAIDS. It is somewhat ironic that these anti-inflammatory agents may themselves produce inflammation in combination with exposure to sunlight. The 2-arylpropionic acids have proven to be the most likely to produce photosensitivity reactions, but other NSAIDS have also been implicated including piroxicam, diclofenac and benzydamine ${ }^{14}$.

Benoxaprofen, since withdrawn due to its hepatotoxicity, was the most photoactive NSAIDS observed. The mechanism of photosensitivity, responsible for the high photo activity of the 2-arylpropionic acids, is the ready decarboxylation of the carboxylic acid moiety. The specific absorbance characteristics of the different chromophoric 2-aryl substituents, affects the decarboxylation mechanism. While ibuprofen is somewhat of an exception, having weak absorption, it has been reported to be a weak photosensitizing agent ${ }^{15}$.

2.1.7. During Pregnancy: NSAIDS are not recommended during pregnancy, particularly during the third trimester. While NSAIDS as a class are not direct teratogens, they may cause premature closure of the fetal ductus arteriosus and renal ADRs in the fetus. Additionally, they are linked with premature birth ${ }^{16}$.

2.1.8. Erectile Dysfunction Risk: A 2005 study linked long term (over 3 months) use of NSAIDS, including ibuprofen, with a 1.4 times increased risk of erectile dysfunction. The report by Kaiser Permanente and published in the Journal of Urology, considered that regular non-steroidal anti-inflammatory drug use is associated with erectile dysfunction beyond what would be expected due to age and other condition ${ }^{17}$.

2.1.9. Other ADRs: Common ADRs, other than listed above, include: raised liver enzymes, headache, and dizziness. Uncommon ADRs include: hyperkalemia, confusion, bronchospasm and rash. Rapid and severe swelling of the the face and body. Ibuprofen may also rarely cause irritable bowel syndrome symptoms. In very rare cases, ibuprofen can cause aseptic meningitis ${ }^{18}$.

2.2. Paracetamol ${ }^{19}$ : Paracetamol is a widely used over-the-counter analgesic (pain reliever) and antipyretic (fever reducer). It is commonly used for the relief of fever, headaches, and other minor aches and pains, and is a major ingredient in numerous cold and flu remedies. In combination with non-steroidal anti-inflammatory drugs (NSAIDS) or opioid analgesics, paracetamol is used also in the management of more severe pain (such as cancer pain).

2.2.1. Adverse Effects: In recommended doses, paracetamol does not irritate the lining of the stomach, affect blood coagulation as much as NSAIDS, or affect function of the kidneys. However, some studies have shown that high dose-usage (greater than 2,000 $\mathrm{mg}$ per day) does increase the risk of upper gastrointestinal 
complications such as stomach bleeding. Paracetamol is safe in pregnancy, and does not affect the closure of the fetal ductus arteriosus as NSAIDS can. Unlike aspirin, it is safe in children, as paracetamol is not associated with a risk of Reye's syndrome in children with viral illnesses ${ }^{\mathbf{2 0}}$.

In 2008 the Lancet published the largest study to date on long term side effects of paracetamol in children. Conducted on over 200,000 children in 31 countries, the study determined that use of paracetamol for fever in the first year of life was associated with a $46 \%$ increase in the risk of developing asthma symptoms when aged 6-7 years. Higher doses and more regular use of the drug are associated with a greater risk of developing asthma; up to a three-fold increase for heavy use. Furthermore, paracetamol use, both in the first year of life and in children aged 6-7 years, was associated with an increased risk of symptoms of rhinoconjunctivitis and eczema. In that article the authors acknowledged that our findings might have been due to confounding by indication, i.e. that the association they found was not causal but rather due to the disease being treated with paracetamol, and emphasized that further research is needed ${ }^{\mathbf{2 1}}$.

Excessive use of paracetamol can damage multiple organs, especially the liver and kidney. In both organs, toxicity from paracetamol is not from the drug itself but from one of its metabolites, $N$-acetyl- $p$-benzoquinoneimine (NAPQI). In the liver, the cyto-chrome P450 enzymes CYP2E1 and CYP3A4 are primarily responsible for the conversion of paracetamol to NAPQI. In the kidney, cyclo-oxygenases are the principal route by which paracetamol is converted to NAPQI. Paracetamol overdose leads to the accumulation of NAPQI, which undergoes conjugation with glutathione. Conjugation depletes glutathione, a natural antioxidant. This in combination with direct cellular injury by NAPQI leads to cell damage and death ${ }^{22}$.

2.3. Opioid analgesic: An opioid is a chemical substance that has morphine like action in the body. The main use is for pain relief. These agents work by binding to opioid receptors, which are found principally in the central nervous system and the gastrointestinal tract. The receptors in these two organ systems mediate both the beneficial effects, and the undesirable side effects. There are a number of broad classes of opioids ${ }^{\mathbf{2 3}}$.

2.3.1. Adverse Reactions in Patient Taking Opioids: These include: Dose-Related respiratory depression, confusion, hallucinations, delirium, urticaria, hypothermia, bradycardia/tachycardia, orthostatic hypotension, dizziness, headache, urinary retention, ureteric or billiary spasm, muscle rigidity, myoclonus and flushing ${ }^{\mathbf{2 4}}$.

2.4. Tramadol $^{25}$ : Tramadol is a CNS depressant and analgesic, used for treating moderate to severe pain. It is a synthetic agent, and it appears to have actions at the $\mu$-opioid receptor as well as the noradrenergic and serotonergic systems. Tramadol was developed by the German pharmaceutical company Grunenthal GmbH in the late 1970s and marketed under the trade name Tramal.

Many other pharmaceutical companies market it under various names. Tramadol is a centrally acting, synthetic analgesic of the aminocyclohexanol group, which has opioid-like effects. It has been in clinical use in Europe since the late 1970s. Its mode of action is not completely understood but it appears to have a dual mechanism of action, which involves inhibition of re-uptake of serotonin (5-HT) and/or nor-adrenaline as well as weak affinity for opioid receptors. Since tramadol does not affect prostaglandin synthesis, it does not have antipyretic or anti-inflammatory effects.

Tramadol has high oral bioavailability. Its absorption is not affected by food. The two oral dosage forms (50mg capsules 4 times daily and 100mg sustained-release tablets twice daily) have been reported to be equivalent in terms of analgesic efficacy and tolerability.

2.4.1. Use ${ }^{26}$ : Publications reporting the clinical use of tramadol are numerous. A number of reviews of the clinical use of tramadol have also been published earlier.

2.4.2. Comparison with Placebo ${ }^{27}$ : A pooled analysis of single-dose, double-blind, randomized, placebocontrolled studies included a total of 1594 patients with post-operative pain following caesarean section or general surgical procedures. Analysis showed that analgesic efficacy of tramadol at doses equal to or greater than $50 \mathrm{mg}$ was superior to that of placebo (statistical value not reported). Likewise, pooled analysis of doubleblind, randomized studies involving 1859 patients with dental extraction pain evaluated single oral doses of tramadol ranging from $50 \mathrm{mg}$ to $200 \mathrm{mg}$. Analysis demonstrated a significantly greater efficacy for tramadol than for placebo at all doses evaluated. A subsequent meta-analysis of these studies confirmed the effectiveness of tramadol in comparison with placebo and demonstrated a significant dose response. Multi-dose studies have also demonstrated a superior effect of oral tramadol over placebo in both pains after dental extraction and pain after orthopedic surgery. 
2.4.3. Comparison with Morphine ${ }^{28}$ : Tramadol (up to 3 doses of 50mg IV) was compared with morphine (up to 3 doses of $5 \mathrm{mg} \mathrm{IV}$ ) over 6 hours in a double-blind, randomized study of 150 patients after gynaecological surgery. In those patients who reported moderate pain, the two drugs were equally effective. However, morphine was superior in patients starting with severe pain. Respiratory depression (as measured by oxygen desaturation) occurred in $13 \%$ of the morphine group but not at all in the tramadol group. Sedation and nausea were also more common in the morphine group.

In a multi-centre, double-blind, randomized study involving 523 patients, the analgesic efficacy of tramadol and morphine given in repeated intravenous boluses as required to control post-operative pain over 24 hours following abdominal surgery was compared. There was no substantive difference in analgesia between tramadol (100mg iv then $100-125 \mathrm{mg}$ IV or IM as needed) and morphine (5mg iv then $5-20 \mathrm{mg}$ IV or IM as needed). The time between the first and second dose of study medication was longer in the tramadol group than in the morphine group, probably due to the lower comparative starting dose of morphine $(5 \mathrm{mg})$ compared with tramadol (100mg). However, the intervals between all subsequent doses were comparable.

Scott et al. reviewed the literature where effectiveness of tramadol in perio-operative pain was assessed using standard visual analogue scales. On the basis of percentage change in pain scores from baseline, they concluded that tramadol effectively relieved moderate to severe postoperative pain associated with several types of surgery, including abdominal, orthopedic and cardiac surgery. Pain scores with tramadol were reduced by approximately 57\% within 4 to 6 hours, compared to a reduction of approximately $70 \%$ with morphine

2.4.4. Comparison with NSAIDS ${ }^{29}$ : In comparative studies of tramadol and ketorolac in nasal surgery and orthopedic surgery, improvements in postoperative analgesia and quality of sleep were similar with intramuscular tramadol or ketorolac.

2.4.5. Comparison with Oral Analgesics ${ }^{30}$ : The analgesic efficacy of single dose, orally administered tramadol $75 \mathrm{mg}$ or $150 \mathrm{mg}$ was compared in a double blind, placebo controlled trial with a combination of paracetamol $650 \mathrm{mg}$ and dextropropoxyphene napsylate $100 \mathrm{mg}$ in 161 patients with severe post operative pain after caesarean section. Both tramadol and the combination analgesic were statistically superior to placebo. Tramadol $150 \mathrm{mg}$ was significantly more effective than both tramadol $75 \mathrm{mg}$ and combination paracetamol/ dextropropoxyphene. No serious adverse effects were observed, but dizziness was more frequently reported with $150 \mathrm{mg}$ tramadol.

On the other hand, Stubhaug compared oral tramadol $50 \mathrm{mg}$ or $100 \mathrm{mg}$ with a combination paracetamol/codeine analgesic $(1000 \mathrm{mg} / 60 \mathrm{mg})$ or placebo in a single-dose study in 144 patients after total hip replacement. In this study the combination of paracetamol/codeine was superior to both doses of tramadol. There was no difference in efficacy between either dose of tramadol and placebo. Adverse effects were more common with tramadol than with the combination, particularly nausea.

Single oral doses of tramadol $75 \mathrm{mg}$ or $150 \mathrm{mg}$ have been compared with codeine phosphate $60 \mathrm{mg}$ and paracetamol/propoxyphene $\mathrm{HCl}(650 \mathrm{mg} / 65 \mathrm{mg})$ in 239 patients with pain after dental extraction. Tramadol $75 \mathrm{mg}$ was more effective than codeine. Although $150 \mathrm{mg}$ of tramadol was superior to the combination analgesic, $75 \mathrm{mg}$ of tramadol was not. In another double-blind, single dose parallel study in 206 patients after dental extraction, tramadol $100 \mathrm{mg}$ was judged to be more effective than codeine $60 \mathrm{mg}$ but only tramadol $200 \mathrm{mg}$ was statistically so.

2.4.6. Pediatrics Surgery ${ }^{31}$ : In pediatric surgery (in patients over the age of 12 months), tramadol has been used effectively for moderate to severe pain in IM or IV doses of $1-2 \mathrm{mg} / \mathrm{kg}$.

2.4.7. Chronic Pain: Tramadol has been included as a step 2 analgesic in the second edition of the World Health Organization's recommendations for treatment of cancer pain. Twenty cancer patients with strong pain unresponsive to previous pain treatment were randomized to receive oral tramadol or morphine solution in a randomized, double-blind, cross-over study. Doses were individually titrated and cross-over occurred on day 4 . Pain scores were similar on day 4, although pain scores were higher in the tramadol group on days 1 and 2. There was a statistically significant reduction in side-effects (particularly nausea and constipation) with tramadol ${ }^{32}$.

Efficacy and safety of high dose oral tramadol (300-600mg/day) compared with low dose oral morphine $(10-60 \mathrm{mg} / \mathrm{day})$ was evaluated in a non-blinded, non-randomized study involving a total of 1658 patients with cancer pain. There was no significant difference in analgesic efficacy between the two groups. Anti-emetic's, laxatives, neuroleptics and steroids were prescribed significantly more frequently in the morphine group; the use of other adjuvants was similar in both groups. Constipation, neuropsychological symptoms and 
pruritus were observed significantly more frequently with low dose morphine. Other symptoms had similar frequencies in both groups.

Oral tramadol was evaluated in a double-blind study over a 4 week period in 390 patients over 65 years of age with chronic pain due to a variety of conditions. Patients were initially treated with either tramadol 50mg orally or paracetamol/codeine $300 \mathrm{mg} / 30 \mathrm{mg}$ and were then allowed to titrate the dose according to pain severity to a total of eight capsules per day. Mean pre-treatment pain intensities were moderate for both groups. At the end of the study, average daily doses were $244 \mathrm{mg}$ of tramadol and 1,407mg/140.7 mg of paracetamol/codeine. Both treatments were rated as good, very good or excellent by $55 \%$ of patients in each group. There was no significant difference in the incidence of adverse effects in each group, but adverse effects resulted in discontinuation in a significantly higher proportion of patients taking tramadol.

Oral tramadol (100mg eighth hourly) was compared with a fixed dose combination of paracetamol/codeine $(1000 \mathrm{mg} / 60 \mathrm{mg}$ eighth hourly) in a randomized, double-blind cross-over study in 55 patients suffering from refractory chronic back pain. Efficacy was similar in the two arms. Although the combination analgesic was reported by the authors as better tolerated than tramadol, the difference was not statistically significant. A multicenter, randomized, double-blind, parallel group study compared the analgesic efficacy and tolerability of immediate release tramadol (50mg four times daily) and sustained-release tramadol (100mg twice daily) in 205 patients with chronic refractory low back pain. There was no difference in pain relief, the course of pain intensity or adverse events between the two groups6. Those continuing beyond the first week became more tolerant of the adverse effects ${ }^{33}$.

The efficacy of tramadol in osteoarthritis has been evaluated in patients who experienced breakthrough pain while being treated with NSAIDS. After an open label phase, forty two patients were randomized to receive tramadol or placebo for a two week period; NSAIDS therapy was continued. Significantly more tramadoltreated patients completed the study. An average daily dosage of $245 \mathrm{mg}$ was significantly more effective than placebo in reducing the severity of pain at rest. In naproxen-responsive patients with painful osteoarthritis of the knee, the addition of tramadol $200 \mathrm{mg} /$ day allowed a significant reduction in NSAIDS dosage without compromising pain relief.

Fifty eight percent were able to discontinue naproxen with the addition of tramadol. Tramadol may have a role in treatment of neuropathic pain of diabetic or other origin and has been estimated to be similar in efficacy to tricyclic antidepressants in such conditions. Tramadol has been reported to be effective across a wide range of chronic pain conditions, including chronic pancreatitis, fibromyalgia, and scleroderma. It should be noted, however, that controlled studies in chronic pain conditions have been of relatively short duration (4-8 weeks) and studies of longer-term use are required ${ }^{34}$.

2.4.8. Adverse Effects: The adverse effects of tramadol are similar to other opioids and include nausea, vomiting, constipation, headache, dizziness, dry mouth, sedation, asthenia, fatigue and sweating. Less common effects include skin reactions and pruritus. Titration of the dose slowly may improve tolerability and intraoperative loading may reduce post-operative nausea and vomiting. With the exception of sweating, constipation and dry mouth, most adverse effects appear to decrease with prolonged use. Tramadol is unlikely to produce clinically relevant respiratory depression at recommended doses but respiratory depression may occur if recommended doses are exceeded. The abuse and/or dependence potential for tramadol are low provided it is dosed within recommended ranges. However, reports of drug dependence and withdrawal have occurred 16. Tramadol has very low affinity for opioid receptors (10 times less than codeine, 60 times less than propoxyphene and 6000 times less than that of morphine)12. Low abuse potential has been confirmed in a randomised, double-blind, placebo-controlled, crossover trial ${ }^{35}$.

\section{Conclusion:}

Tramadol has a dual mechanism of action that explains its effectiveness in those types of pain that are refectory to conventional opioids and its better tolerability profile. At therapeutic doses, Tramadol has weak/no effects on respiration, cardiovascular system and intestinal motility. Compared with traditional NSAIDS, Tramadol lacks serious GI renal toxicity, which is a major concern in the elderly. These side effects are generally mediated by prostaglandins and so are not seen with Tramadol. Even though the new COX-2 specific NSAIDS may demonstrate greater GI safety than the older NSAIDS, Tramadol has a superior safety profile. Hepato-toxicity considers being a class characteristic of NSAIDS that has not been reported to date with Tramadol. Unlike acetaminophen, Tramadol has no antipyretic activity. So it does not mask any possible infective and/or phlogistic processes. Tramadol can be used safely even in patients with hypertension, congestive heart failure, or renal insufficiency, because such patient such patients may not tolerate the renal side effects of NSAIDS. It is safer in patients with asthma and GI ulcers. Tramadol has every low affinity for opioid receptors. Consequently, constipation and respiratory depression are less frequent with Tramadol at therapeutic doses as compared to conventional opioids. There is also less risk of abuse and dependence with Tramadol and 
may result in lack of compliance. Noncompliance with high - frequency dosing regimens results in inconsistent analgesia. Tramadol represents a solution to the unfavorable side effects profiles of other analgesic typically used for unrelieved chronic pain.

\section{References:}

[1]. Kowaluk E A, Arneric S P, Williams M. Opportunities in pain therapy beyond the opioids and NSAIDS. Emerging Drugs. 1998; 3: $1-37$.

[2]. Strong J, Unruh A M, Wright A. Pain a textbook for therapists. 2nd ed. UK: Harcourt publisher; 2003. p. 4 - 5.

[3]. Marco P. The neurological basis of pain. $1^{\text {st }}$ ed. USA: McGraw Hills; 2005. p. 3 - 4.

[4]. Paul FS, Epstein JB. Oral complication and its management. New York: Oxford University press; 2010. p. 246 - 48.

[5]. Lemke T L, Williams DA, Roche V, Zito W. Principles of medicinal chemistry. $6^{\text {th }}$ ed. Philadelphia: Liincots Williams and wilkins; 2010. p. 954 - 55.

[6]. Green G. Understanding NSAIDS from aspirin to COX-2. Clinical Cornerstone. 2001; 3(5): 50 - 60

[7]. Kearney P, Baigent C, Godwin J, Halls H, Emberson J, Patrono C. Do selective cyclo-oxygenase-2 inhibitors and traditional non steroidal anti-inflammatory drugs increase the risk of atherothrombosis, meta-analysis of randomized trials? BMJ. 2006; 332(7553): $1302-08$.

[8]. Page J, Henry D. Consumption of NSAIDS and the development of congestive heart failure in elderly patients: an under recognized public health problem. Archives of Internal Medicine. 2000; 160(6): $777-84$.

[9]. Traversa G, Walker A, Iolito F, Caffari B, Capurso L, Dezi A, Koch M, Maggini M, Alegiani S, Raschetti R. Gastroduodenal toxicity of different non steroidal anti inflammatory drugs. Epidemiology Cambridge Mass. 1995; 6 1: 49 - 54.

[10]. Pincus T, Griffin M. Gastrointestinal disease associated with non steroidal anti-inflammatory drugs new insights from observational studies and functional status questionnaires. Am. J. Medicine. 1991; 91(3): $209-12$.

[11]. Fries J F, Williams CA, Bloch DA, Michel BA. Non steroidal anti-inflammatory drug- associated gastropathy incidence and risk factor models. Am. J. Medicine. 1991; 91 (3): $213-22$.

[12]. Dunn MJ, Simonson M, Davidson E W, Scharschmidt L A, Seditionor J R. Non steroidal anti-inflammatory drugs and renal function. J. Clin. Pharmacol. 1988; 28(6): $524-29$.

[13]. Murray M D and Brater D C. Adverse effects of non steroidal anti-inflammatory drugs on renal function. Ann. Intern. Medicine. 1990; 112 (8): $559-60$

[14]. Thomas M Diuretics ACE inhibitors and NSAIDS -the triple whammy. The Medical Journal of Australia 2006; 172 (4): $184-185$

[15]. Sandler D P, Burr F R, Weinberg C R. Non steroidal anti-inflammatory drugs and the risk for chronic renal disease. Ann Intern Medicine. $1991 ; 115(3): 165-72$.

[16]. Moore D. Drug-induced cutaneous photosensitivity incidence, mechanism, prevention and management, Drug safety. International Journal of Medical Toxicology and Drug Experience. 2002; 25 (5): 345 - 72.

[17]. Tisdale J E and Douglas A M. Drug-Induced Diseases Prevention, Detection and Management. $2^{\text {nd }}$ ed. American society of health system pharmacist; 2010. p. 122 - 26

[18]. Ostensen M and Skomsvoll J Anti-inflammatory pharmacotherapy during pregnancy. Expert Opinion on Pharmacotherapy. 2004; 5 (3): $571-80$.

[19]. Hamid R N P, Perrine B, Odile S and Anick B Use of non aspirin non steroidal anti-inflammatory drugs during pregnancy and the risk of spontaneous abortion. Canadian Medical Association Journal. 2011; 183(15): 1713 - 20

[20]. Nielsen G L, Sorensen H T, Larsen H and Peditionersen L: Risk of adverse birth outcome and miscarriage in pregnant users of nonsteroidal anti-inflammatory drugs population based observational study and case-control study .BMJ. 2001; 322 (7281): 266 - 70.

[21]. Shiri. Effect of Non steroidal Anti-Inflammatory Drug Use on the Incidence of Erectile Dysfunction. Journal of Urology. 2005 ; 175 (5): $1812-16$

[22]. Akil M, Amos R S and Stewart P. Infertility may sometimes be associated with NSAIDS consumption. Br. J. Rheumatol. 1996; 35 (1): $76-78$.

[23]. Joseph M, Gleason J M, Slezak J, Howard K, Kristi R, Stephen K, Eeditionen V D, Haque R, Virginia P, Ronald K and Steven J. Regular Non steroidal Anti-Inflammatory Drug Use and Erectile Dysfunction. The Journal of Urology. $2011 ; 185$ (4): 1388 - 93.

[24]. Fowler PD. Aspirin paracetamol and non-steroidal anti-inflammatory drugs a comparative review of side effects. Medicine. Toxicol. Adverse Drug Exp. 1987; 2 (5): 338 - 66.

[25]. Borne R, Levi M, Wilson N. Foye's Principle of Medicinal chemistry. $6^{\text {th }}$ ed. New Delhi: Lipincott williams and wilkins; 2008. p. $963-65$.

[26]. Scottish Intercollegiate Guidelines Network. Guideline 106, Control of pain in adults with cancer, Scotland National Health Service, 2008.

[27]. Bronwen B, Knights K, Bryant BJ, Knights KM, Salerno E and Knights B. Pharmacology for Health Professionals. $3^{\text {rd }}$ ed. Australia: Elsevier, 2011. p. 300 .

[28]. Seditionigheh E, Esfahani S A, Ghaffarian H R, Khoshneviszade M. Comparison of efficacy and safety of acetaminophen and ibuprofen administration as single dose to reduce fever in children. Iranian Journal of Pediatrics. $2010 ; 20$ (4): 500 - 1.

[29]. Lesko S M, Mitchell A. The safety of acetaminophen and ibuprofen among children younger than two years old. Pediatrics. 1999; 104 (4): 39.

[30]. Beasley R. Association between paracetamol use in Infancy and childhood and risk of asthma, rhino conjunctivitis and eczema in Children. Lancet. 2008; 372: 1039 - 1048.

[31]. Calder I C, Creek M J, Williams P J. N-hydroxylation of p-acetophenetidide as a factor in nephron toxicity. J. Medicine. Chem. 1973; 16: $499-502$.

[32]. Smil kstein M J, Kna GL, Kulig KW. Efficacy of oral N-acetyl cysteine in the treatment of acetaminophen overdose, Analysis of the national multi center study from 1976 t o 1985. N. Engl. J. Medicine. 1988; 319:1557 - 562.

[33]. Hughes J, Smith T W, Kosterlitz H W. Identification of two related penta peptides from the brain with potent opiate agonist activity. Nature. 1975; 258: $577-79$.

[34]. Lord J H, Water A and Hughes J. Endogenous opioid peptides multiple agonists and receptors. Nature. $1977 ; 267: 495$ - 99.

[35]. Ramsin B, Andrea M T, Datta S, Buenaventura R, Adlaka R, Sehgal N, Glaser S E, Vallejo R. Opioid Complications and Side Effects. Pain Physician. 2008; 11: 105 - 120 